\title{
Legged Fire Fighter Robot Movement Using PID
}

\author{
Abdul Latif ${ }^{1}$, K. Shankar ${ }^{2}$, Phong Thanh Nguyen ${ }^{3}$ \\ ${ }^{1}$ School of Mathematics, Physics and Technology, Universitas Islam Sultan Agung Semarang, Semarang, Indonesia \\ ${ }^{2}$ Department of Computer Applications, Alagappa University, Karaikudi, India. \\ ${ }^{3}$ Department of Project Management, Ho Chi Minh City Open University, Vietnam. \\ Email: ${ }^{1}$ 4bdullatif.u.m.y@gmail.com, ${ }^{2}$ shankarcrypto@gmail.com, ${ }^{3}$ phong.nt@ou.edu.vn
}

\begin{abstract}
Proportional Integral Derivative (PID) control is a control system commonly used by industry. Approximately 90\% of industrial equipment uses a PID controller because it is easy to use. In the Indonesian Fire Extinguisher Robot Contest (IFERC), the contested robots must follow the contours of the walls of the arena. A Fire extinguisher robot navigation was chosen because the race arena of the competition consisted of walls with different aisles and rooms. The navigation robots used PID control. This study designed and implemented a control algorithm for legged fire extinguishing robots using the PID method, where the PID control was processed in a microcontroller. The angles for each servo motor generated by the calculation of the PID enable the robot to navigate by taking decisions to move quickly or slowly, turn right, turn left and stop. The robot's proximity sensor data and fire sensors enable the fire to be extinguished. The result showed that the robot can carry out its duties optimally.
\end{abstract}

Keywords-Microcontroller, PID control, Proximity sensor, robot navigation system

\section{INTRODUCTION}

Human activities relating to technology have been increasing rapidly in the modern era. To support the increasingly high needs of life, people surely will work very hard. Therefore, they have created a robot to help and replace human performance to facilitate their works.

A robot is a combination of mechanical, electronic and computer science. Robot construction, in general, has an actuator in the form of a wheel or leg to move the entire robot body, so that the robot can move position from one point to another.

To bridge the development of robot technology and student creativity containers, the Indonesian DirectorateGeneral for Higher Education has been holding several robot contests. One of them is the annual Indonesian Robot Contest (KRI) consisting of four categories, namely The Indonesian Robot Contest (KRI), the RoboSoccer Humanoid League Contest, the Indonesian Art Robot Contest (KRSI) and the Indonesian Fire Extinguisher Robot Contest (KRPAI).

In this research, the method applied was by combing the walls in the arena using the SRF-05 ultrasonic sensor to detect distances. It needs a control system to overcome the problems in the movement.
Some researchers have conducted research on robot navigation. Crepon, Panchea, and Chapoutot implemented reliable navigation planning on two-wheeled mobile robots [1]. The navigation system used the Rapidly-exploring Random Tree (RRT) algorithm. Han studied sensor-based cellular robot navigation through strengthening learning [2]. The navigation system used a deep learning algorithm. Morad, Kalita, and Thangavelautham investigated planning and navigation of climbing robots in low gravity environments was, [3]. The navigation system used A*algorithm. Mikhaylov and Lositskii examined forest robots' control and navigation [4] The navigation system used a neural network algorithm.

Adamov investigated the effect of the Mecanum wheel construction on the accuracy of omnidirectional platform navigation (for example the KUKA youBot robot) [5]. The navigation system used Odometric navigation. Baslan studied the navigation, guidance, and control of free-flying robots during their encounters with passive space vehicles [6]. The navigation system used the Nearness Diagrams algorithm. Kumar Vamossy investigated laser scan matching in robot navigation [7]. The navigation system used a neural network algorithm. Socially Conscious Robot Navigation Using Remote Strengthening Learning was investigated by Xuan Tung and Dung Ngo [8]. The navigation system used the Deep Reinforcement Learning algorithm.

Research on autonomous navigation of mobile robots based on multi-fusion ultrasonic sensors was conducted by Wang [9]. The navigation system used ultrasonic sensors to detect objects. Bobkov studied autonomous underwater robot image-based navigation and reconstruction of the $3 \mathrm{D}$ environment [10]. The navigation system used the camera to detect obstacles in front of it. Research on the intelligent fuzzy controller for human-conscious robot navigation was conducted by Obo and Yasuda [11], while on geometrical optical analysis of the augmented reality augmented projection system for robot navigation was conducted by Tsujimura, Aoki, and Izumi [12]. An intelligent fuzzy controller and geometrical optics algorithms were used for robot navigation respectively.

ByungSoo Ko investigated neural network-based autonomous navigation for homecare mobile robots [13]. The navigation system used a neural network algorithm. Adib and Masoumi investigated cellular robots' navigation in unknown 
environments using fuzzy logic and learning automata [14]. The navigation system used a fuzzy algorithm and automata. Savkin and Wang studied a method for collision-free navigation of non-holonomic 3D robots in unknown environments such as tunnels [15]. The 3D navigation algorithm was used for robot navigation. Guo designed a robotic localization and multi-vision navigation system service [16]. The system used the SLAM visual algorithm.

Guoqing and Tao examined a bio-inspired autonomous navigation system for logistical mobile robots with inertial AHRS [17]. The navigation system used a navigation control algorithm namely attitude and heading reference systems (AHRS). Aissa, Fatima, and Yassine examined data fusion strategies for navigating cellular robots in unknown environments using fuzzy logic controls, [18]. The navigation system used a fuzzy algorithm. Li and Savkin investigated navigation algorithms for non-holonomic cellular robots that are navigated by sensor networks in a messy and dynamic environment [19]. A discrete-time navigation algorithm was used to avoid obstacles. Research on the Rear Navigation Navigation Projection Method Based on the Bi-plane Calibration Method was conducted by Shiyi [20]. The biplanning algorithm was used to avoid obstacles and get to the destination.

The KRPAI Robot Legged Division Navigation System was designed in this study using a PID Controller. The design of this PID controller used hand-tuning to determine the size of $\mathrm{KP}, \mathrm{Ki}$, and $\mathrm{Kd}$. The control was performed by determining the set-point value in the form of the desired distance to the wall. Furthermore, the distance data were processed using a controller that produces a signal to control the robot's movement so that it could determine the robot's distance to the wall. Then the output went through a feedback process where the error was indicated by the difference between the input and the output response. After that, the parameters of the PID controller were determined so that the close loop system met the desired performance criteria.

\section{METHOD}

\section{A. Hardware Design}

The first step of the hardware design was to create or design a model for the movement of a legged fire extinguisher robot using the Proportional Integral Derivative (PID) method [21]-[24].The movement system of the legged fire extinguisher robot using the method (PID) to be used.

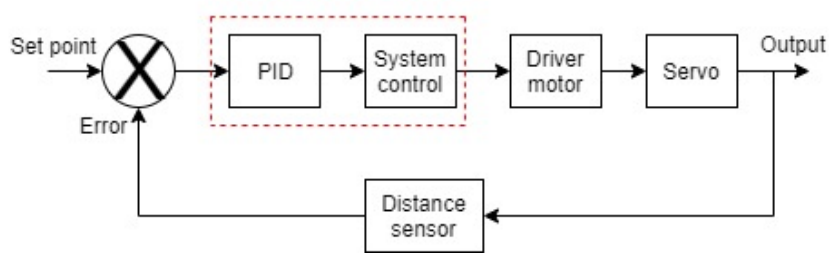

Fig. 1. PID system block diagram

The ultrasonic sensor was the main sensor used in this study. The sensor was a proximity sensor to keep the distance between the robot body and the wall/obstruction not to crash [25]-[29]. The results of the sensor readings were sent to the STM32 microcontroller [30]. The microcontroller processed the results from the proximity sensor and sent them to the LCD in the form of text. The servo was the actuator used to move the robot from one point to another.

\section{B. Firmware Design}

The PID controller aimed to adjust the robot servo degrees when walking along the walls of the race arena. The determination of the results of the PID controller parameters was obtained by using the hand-tuning method. The results obtained from the PID controller values were $\mathrm{Kp}, \mathrm{Ki}$, and $\mathrm{Kd}$.

The system block diagram and the PID system block diagram was made into a single unit in a flowchart to make it easy to analyze the workings of the entire system on this tool. The following is a description of the flow chart.

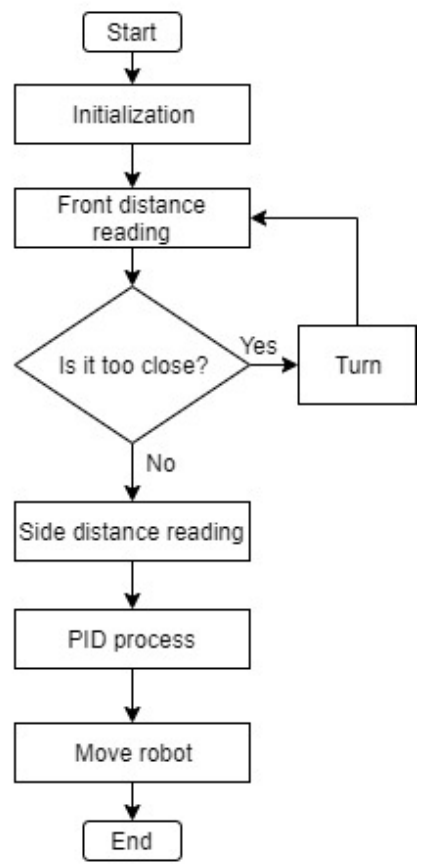

Fig. 2. System workflow

The robot motion process using the PID method began when the device was on. An ultrasonic sensor is a device that measures the distance between the body of a robot and the wall. When the start button was pressed, the sensor started to read the distance to the object. The input from the proximity sensor reading was sent to the STM32 microcontroller. After that, the results of the PID obtained by reading the proximity sensor were calculated and transformed into degrees sent to the servo.

If the proximity sensor is less than the set-point value, the robot will turn, but if the distance sensor value exceeds the set-point value, the robot will move forward. If the reading of the side distance sensor exceeds the set-point value, the robot will move away from the object and vice versa. If the side distance sensor reading is less than the set-point value, the robot will approach the object to keep the distance so that the robot remains within the set-point range.

\section{Mechanical Design}

The layout of the robot body clarified the positioning of sensors and the location of other components. The next step 
was the positioning of the proximity sensor and the front leg robot.

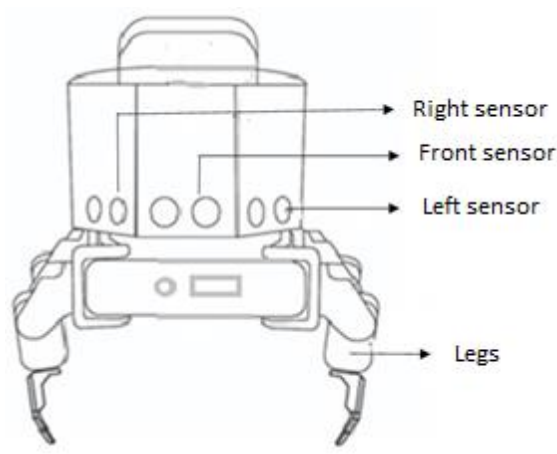

Fig. 3. Visualization of the front robot

Figure 3 shows that the SRF-05 proximity sensor is the main navigation sensor for this legged fire extinguisher robot to maintain the distance between the robot and the wall. The robot is called a quadruped robot with 2 front legs and 2 rear legs.

Five SRF-05 ultrasonic proximity sensors were used to keep the robot away from the wall. The LCD was used to display information from the sensors and there were several buttons to set the desired PID values. And they were all managed together using an STM32 microcontroller.

\section{PID Design and Configuration}

The design of the PID method used was the ultrasonic sensor distance value and the setpoint value in the PID control tuning process.

Making this PID controller program is based on the digital PID controller equation. A digital PID controller is another form of PID controller that is programmed and run using a computer or microcontroller. Error and last_error that will be used in the PID controller action calculation. Every time a program is looped, the error will be updated with data taken from the sensor, and previously it will be saved in last_error. The output of the PID controller program calculation is the angle value to drive each servo motor.

Equations (1) through (4) can be shown from the values of the variables as follow,

$$
\begin{aligned}
& u(t)=K_{P} e(k)+K_{i} T \sum_{0}^{k} e(k)+\frac{1}{T} K_{d}\left(e_{k}-e_{k-1}\right) \\
& \text { Front and right back servos = centre angle }+ \text { PID } \\
& \text { Front and left back servos }=\text { centre angle }- \text { PID }
\end{aligned}
$$

Setpoint (SP) is a parameter of a reference value or desired value. Present Value (PV) is the measured sensor reading value which is then stored to a variable, or measured variable that is fed back by the sensor (feedback signal from the sensor). Tuning parameters work to set the PID parameters $(\mathrm{Kp}, \mathrm{Ki}$, and $\mathrm{Kd})$ as well as other parameters such as time sampling, setpoint determination, etc.

\section{IMPLEMENTATION}

The tests were performed to know the performance of the distance sensor and the robot.

\section{A. Distance sensor test}

To assess the distance between the robot and the wall, the SRF-05 proximity sensor was used. An observation using the SRF-05 proximity sensor was made using the STM32 microcontroller pin. The following is the observations of the proximity sensor SRF-05.

TABLE I. COMPARISON TEST RESULT FOR SRF-05

\begin{tabular}{|c|c|c|c|c|}
\hline No & $\begin{array}{c}\text { Real } \\
\text { distance } \\
(\mathbf{c m})\end{array}$ & $\begin{array}{c}\text { Distance } \\
\text { sensor } \\
(\mathbf{c m})\end{array}$ & $\begin{array}{c}\text { Error } \\
(\%)\end{array}$ & $\begin{array}{c}\text { Accuracy } \\
(\%)\end{array}$ \\
\hline 1 & 12 & 12.4 & 3.33 & 96.67 \\
\hline 2 & 20 & 20.6 & 3 & 97 \\
\hline 3 & 50 & 50.2 & 0.4 & 99.6 \\
\hline 4 & $\mathbf{8 0}$ & $\mathbf{8 0 . 2}$ & $\mathbf{0 . 2 5}$ & $\mathbf{9 9 . 7 5}$ \\
\hline 5 & 100 & $\mathbf{9 9 . 8}$ & $\mathbf{0 . 2}$ & $\mathbf{9 9 . 8}$ \\
\hline
\end{tabular}

\section{$B$. Overall system test}

The overall system test was carried out to determine the performance of the robotic PID controller based on the Kp, $\mathrm{Ki}$ and $\mathrm{Kd}$ parameters obtained from the robotic motion observations in the Hallway area. In this experiment, the robot will follow the right side of the track wall.

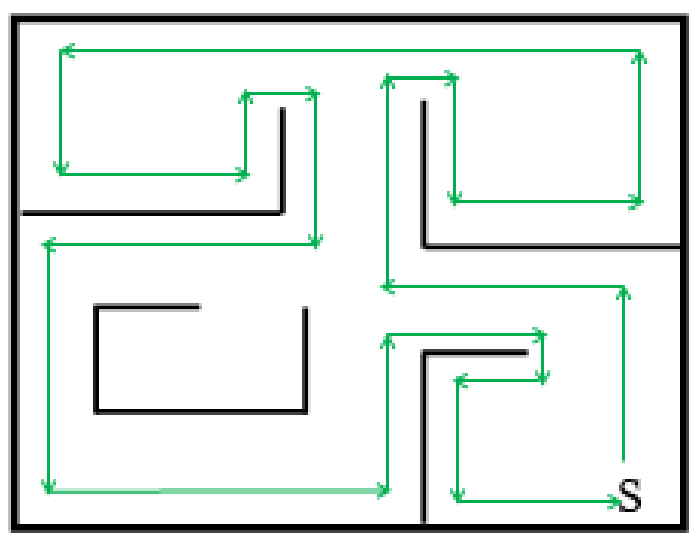

Fig. 4. Track to test the robot

On the track, the Robot is placed in the initial position (S). The arrow direction shows an illustration of the route the robot took during the testing process. Table 2 presents the test results with $\mathrm{P}=5, \mathrm{I}=3$, and $\mathrm{D}=2$ PID values and set-point $12 \mathrm{~cm}$.

TABLE II. TEST RESULT OF THE ROBOT TO FOLLOW THE RIGHT WALL.

\begin{tabular}{|c|c|c|l|}
\hline Test No & Amount of collision & Time (s) & Result \\
\hline 1 & 2 & 40 & Success \\
\hline 2 & 1 & 50 & Success \\
\hline 3 & 0 & 60 & Success \\
\hline 4 & $\mathbf{0}$ & $\mathbf{7 0}$ & Success \\
\hline 5 & $\mathbf{1}$ & $\mathbf{8 0}$ & Success \\
\hline
\end{tabular}

\section{CONCLUSION}

Several conclusions can be drawn from the results of the research carried out in the course of this study, including the SRF-05 ultrasonic sensor has a high degree of accuracy in reading distance from objects in the form of walls with an average error of $1,436 \mathrm{~cm}$. The use of the PID controller on the firefighting robot has made the robot's movements very balanced and able to make the robot run smoothly. The robot 
moves to follow the right wall perfectly, even though the robot sometimes collides. When assigning values to the PID, a perfect value was not found when the setpoint value was changed.

\section{REFERENCES}

[1] P.-A. Crepon, A. M. Panchea, and A. Chapoutot, "Reliable Navigation Planning Implementation on a Two-Wheeled Mobile Robot," in 2018 Second IEEE International Conference on Robotic Computing (IRC), 2018, vol. 2018-Janua, no. Line 1, pp. 173-174.

[2] S.-H. Han, H.-J. Choi, P. Benz, and J. Loaiciga, "Sensor-Based Mobile Robot Navigation via Deep Reinforcement Learning," in 2018 IEEE International Conference on Big Data and Smart Computing (BigComp), 2018, pp. 147-154.

[3] S. Morad, H. Kalita, and J. Thangavelautham, "Planning and navigation of climbing robots in low-gravity environments," in 2018 IEEE/ION Position, Location and Navigation Symposium (PLANS), 2018, pp. 1302-1310.

[4] M. N. Mikhaylov and I. A. Lositskii, "Control and navigation of forest robot," in 2018 25th Saint Petersburg International Conference on Integrated Navigation Systems (ICINS), 2018, pp. 12.

[5] B. I. Adamov, "Influence of mecanum wheels construction on accuracy of the omnidirectional platform navigation (on exanple of KUKA youBot robot)," in 2018 25th Saint Petersburg International Conference on Integrated Navigation Systems (ICINS), 2018, pp. 14.

[6] N. Baslan, S. Heerklotz, S. Weber, A. Heerklotz, B. Hofig, and J. Abu-Khalaf, "Navigation and Vision System of a Mobile Robot," in 2018 19th International Conference on Research and Education in Mechatronics (REM), 2018, pp. 99-104.

[7] N. Kumar and Z. Vamossy, "Laser Scan Matching in Robot Navigation," in 2018 IEEE 12th International Symposium on Applied Computational Intelligence and Informatics (SACI), 2018, no. 6, pp. 000241-000246.

[8] T. Xuan Tung and T. Dung Ngo, "Socially Aware Robot Navigation Using Deep Reinforcement Learning," in 2018 IEEE Canadian Conference on Electrical \& Computer Engineering (CCECE), 2018, vol. 2018-May, pp. 1-5.

[9] R. Wang, L. Chen, J. Wang, P. Zhang, Q. Tan, and D. Pan, "Research on autonomous navigation of mobile robot based on multi ultrasonic sensor fusion," in 2018 IEEE 4th Information Technology and Mechatronics Engineering Conference (ITOEC), 2018, no. Itoec, pp. 720-725.

[10] V. A. Bobkov, A. P. Kudryashov, S. V. Melman, and V. P. May, "Image-Based Navigation of Autonomous Underwater Robot and 3D Reconstruction of Environment," in 2018 3rd Russian-Pacific Conference on Computer Technology and Applications (RPC), 2018, pp. 1-4.

[11] T. Obo and E. Yasuda, "Intelligent Fuzzy Controller for HumanAware Robot Navigation," in 2018 12th France-Japan and 10th Europe-Asia Congress on Mechatronics, 2018, pp. 392-397.

[12] T. Tsujimura, R. Aoki, and K. Izumi, "Geometrical Optics Analysis of Projected-Marker Augmented Reality System for Robot Navigation," in 2018 12th France-Japan and 10th Europe-Asia Congress on Mechatronics, 2018, pp. 1-6.

[13] ByungSoo Ko, Ho-Jin Choi, C. Hong, J.-H. Kim, Oh Chul Kwon, and C. D. Yoo, "Neural network-based autonomous navigation for a homecare mobile robot," in 2017 IEEE International Conference on Big Data and Smart Computing (BigComp), 2017, pp. 403-406.

[14] A. Adib and B. Masoumi, "Mobile robots navigation in unknown environments by using fuzzy logic and learning automata," in 2017 Artificial Intelligence and Robotics (IRANOPEN), 2017, pp. 58-63.

[15] A. V. Savkin and C. Wang, "A method for collision free navigation of non-holonomic 3D robots in unknown tunnel like environments," in 2017 IEEE International Conference on Robotics and Biomimetics (ROBIO), 2017, vol. 2018-Janua, no. 4, pp. 936-940.

[16] J. Guo, X. Xiao, P. Pan, and X. Luo, "A design of multi-vision localization and navigation service robot system," in 2017 12th International Conference on Computer Science and Education (ICCSE), 2017, no. Iccse, pp. 787-790.

[17] Z. Guoqing and L. Tao, "Bio-inspired autonomous navigation system for logistics mobile robots with inertial AHRS," in 2017 IEEE 3rd Information Technology and Mechatronics Engineering Conference (ITOEC), 2017, vol. 2017-Janua, pp. 971-975.

[18] B. Aissa, C. Fatima, and A. Yassine, "Data fusion strategy for the navigation of a mobile robot in an unknown environment using fuzzy logic control," in 2017 5th International Conference on Electrical Engineering - Boumerdes (ICEE-B), 2017, vol. 2017Janua, pp. 1-6.

[19] H. Li and A. V. Savkin, "A navigation algorithm for a nonholonomic mobile robot navigated by a sensor network in dynamic cluttered environments," in 2017 36th Chinese Control Conference (CCC), 2017, pp. 5801-5804.

[20] Y. Shiyi, L. Song, M. Wenhua, Z. Fengfeng, and S. Lining, "Research on Back Projection Method of Robot Navigation Based on Bi-plane Calibration Method," in 2017 IEEE 7th Annual International Conference on CYBER Technology in Automation, Control, and Intelligent Systems (CYBER), 2017, pp. 1220-1223.

[21] C. H. KAZANCI and A. F. KOCAMAZ, "PID Optimization on Differential Drive Mobile Robot," in 2018 International Conference on Artificial Intelligence and Data Processing (IDAP), 2018, pp. 16.

[22] Y. Li, Z. Li, and Y. Wang, "Quadrotor Drift Error Correction Based on Adaptive PID," in 2018 Chinese Automation Congress (CAC), 2018, pp. 446-451.

[23] J. Gomez-Avila, C. Lopez-Franco, A. Y. Alanis, and N. AranaDaniel, "Control of Quadrotor using a Neural Network based PID," in 2018 IEEE Latin American Conference on Computational Intelligence (LA-CCI), 2018, no. 1, pp. 1-6.

[24] E. Malayjerdi, H. Kalani, and M. Malayjerdi, "Self-Tuning Fuzzy PID Control of a Four-Mecanum Wheel Omni-directional Mobile Platform," in Electrical Engineering (ICEE), Iranian Conference on, 2018, pp. 816-820.

[25] A. M. Zaki, O. Arafa, and S. I. Amer, "Microcontroller-based mobile robot positioning and obstacle avoidance," J. Electr. Syst. Inf. Technol., vol. 1, no. 1, pp. 58-71, May 2014.

[26] R. Palm and D. Driankov, "Fluid Mechanics for Path Planning and Obstacle Avoidance of Mobile Robots," in Proceedings of the 11th International Conference on Informatics in Control, Automation and Robotics, 2014, vol. 2, pp. 231-238.

[27] R. Kumar et al., "Maze Solving Robot with Automated OAvoidance," Procedia Comput. Sci., vol. 105, no. December 2016, pp. 57-61, 2017.

[28] A. O. Adeodu, I. A. Daniyan, T. S. Ebimoghan, and S. O. Akinola, "Development of an Embedded Obstacle Avoidance and Path Planning Autonomous Solar Grass Cutting Robot for SemiStructured Outdoor Environment," in 2018 IEEE 7th International Conference on Adaptive Science \& Technology (ICAST), 2018, vol. 2018-Augus, pp. 1-11.

[29] I. W. S. Nirawana, K. Y. E. Aryanto, and G. Indrawan, "Mobile Robot Based Autonomous Selection of Fuzzy-PID Behavior and Visual Odometry for Navigation and Avoiding Barriers in the Plant Environment," in 2018 International Conference on Computer Engineering, Network and Intelligent Multimedia (CENIM), 2018, no. 2, pp. 234-239.

[30] S. Guo, Z. Wang, J. Guo, Q. Fu, and N. Li, "Design of the Speech Control System for a Upper Limb Rehabilitation Robot Based on Wavelet De-noising," in 2018 IEEE International Conference on Mechatronics and Automation (ICMA), 2018, pp. 2300-2305. 